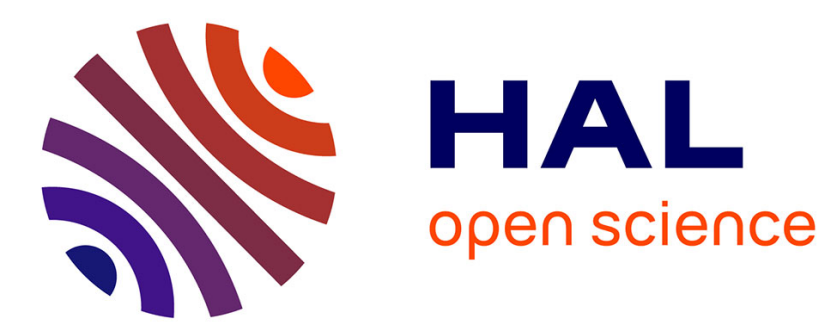

\title{
Online corrective responses following target jump in altered gravitoinertial force field point to nested feedforward and feedback control
}

Loïc Chomienne, Jean Blouin, Lionel Bringoux

\section{- To cite this version:}

Loïc Chomienne, Jean Blouin, Lionel Bringoux. Online corrective responses following target jump in altered gravitoinertial force field point to nested feedforward and feedback control. Journal of Neurophysiology, 2020, 10.1152/jn.00268.2020 . hal-03011323

\section{HAL Id: hal-03011323 \\ https://hal-amu.archives-ouvertes.fr/hal-03011323}

Submitted on 18 Nov 2020

HAL is a multi-disciplinary open access archive for the deposit and dissemination of scientific research documents, whether they are published or not. The documents may come from teaching and research institutions in France or abroad, or from public or private research centers.
L'archive ouverte pluridisciplinaire HAL, est destinée au dépôt et à la diffusion de documents scientifiques de niveau recherche, publiés ou non, émanant des établissements d'enseignement et de recherche français ou étrangers, des laboratoires publics ou privés. 
1 Online corrective responses following target jump in altered gravitoinertial force

2 field point to nested feedforward and feedback control

$4 \quad{ }^{1}$ Aix Marseille Univ, CNRS, ISM, Marseille, France

$5 \quad{ }^{2}$ Aix-Marseille Univ, CNRS, LNC, Marseille, France

6 *Correspondence:

7 Dr. Lionel Bringoux

8 lionel.bringoux@univ-amu.fr

9 UMR 7287 CNRS \& Aix-Marseille Université - Faculty of Sport Science

10163 Avenue de Luminy, CP 910 - 13288 Marseille cedex 9 


\section{Abstract}

Studies on goal-directed arm movements have shown a close link between feedforward and feedback control in protocols where both planning and online control processes faced a similar type of perturbation, either mechanical or visual. This particular context might have facilitated the use of an adapted internal model by feedforward and feedback control. Here we considered this link in a context where after feedforward control was adapted through proprioception-based processes, feedback control was tested under visual perturbation. We analyzed the response of the reaching hand to target displacements following adaptation to an altered force field induced by rotating participants at constant velocity. Reaching corrections were assessed through variables related to the accuracy (lateral and longitudinal endpoint errors) and kinematics (movement time, peak velocity) of the corrective movements. The electromyographic activity of different arm muscles (pectoralis, deltoid, biceps and triceps brachii) was analyzed. Statistical analyses revealed that accuracy and kinematics of corrective movements were strikingly alike between normal and altered gravitoinertial force fields. However, pectoralis and biceps muscle activities recorded during corrective movements were significantly modified to counteract the effect of rotation-induced Coriolis and centrifugal forces on the arm. Remarkably, feedback control was functional from the very first time participants encountered a target jump in the altered force field. Overall, the present results demonstrate that feedforward control enables immediate functional feedback control even when applied to distinct sensorimotor processes. 
Motor responses to target jump in altered force field

\section{New \& Noteworthy}

We investigated the link between feedforward and feedback control when applying a

35 double-step perturbation (visual target jump) during reaching movements performed in 36 modified gravitoinertial environments. Altogether, kinematics and EMG analyses showed that

37 movements corrections were highly effective in the different force fields suggesting that, 38 although feedforward and feedback control were driven by different sensory inputs, feedback 39 control was remarkably functional, from the very first time participants encountered a target 40 jump in the altered force field.

41 Running title:

42 Motor responses to target jump in altered force field

\section{Keywords:}

45 motor control; reaching movement; force field adaptation; double-step paradigm; internal model 
Motor responses to target jump in altered force field

\section{Introduction}

Catching an object slipping from our moving hands before it hits the ground reflects the astonishing ability of feedback control to deal with unpredictable perturbations though online corrective processes. The question remains as to whether, and if so how, such corrective motor responses are readily functional after adaptation of the feedforward control responsible for triggering arm motor commands. We addressed this issue by studying arm-reaching motor responses to unforeseen changes in target position following sustained exposure to an altered gravitoinertial force field.

Several parameters must be taken into account to produce motor commands for intended motor actions in stable environments. For instance, the initial position of the hand (Rossetti et al. 1995; Vindras et al. 1998), movement extent and direction (Messier and Kalaska 2000; Riehle and Requin 1989; Sarlegna and Blouin 2010), movement velocity (Churchland et al. 2006; Moran and Schwartz 1999) and the effect of gravity on the arm (Gaveau et al. 2016; Papaxanthis et al. 1998) are key parameters in preparing motor commands. Set before movement onset, these parameters are thought to be under feedforward control (Desmurget and Grafton 2000). Importantly, the feedforward control can adapt to internal (e.g., growth) or external (e.g., force field) changes that persist in time. In the case of a change of the gravitoinertial force field, this adaptation would rely on internal models updating of arm dynamics and environmental properties enabled by feedback errors processing (Shadmehr 2004). Thanks to this sensorimotor adaptation, which greatly rely on the cerebellar network (Donchin et al. 2012; Maschke et al. 2004), the motor actions performed in the new gravitational environment become comparable to those produced in the normal force field (Coello et al. 1996; Lackner and Dizio 1994; Sarlegna et al. 2010). 
On the other hand, when planning errors occur or when movement planning is no longer valid due to sudden and unpredictable perturbations, feedback control allows the ongoing movement to be corrected accordingly. This capacity has been demonstrated by studies showing that participants could still produce accurate goal-directed arm movements even when targets suddenly changed position after movement onset (Day and Lyon 2000; Desmurget et al. 1999; Pélisson et al. 1986; Sarlegna et al. 2003; Soechting and Lacquaniti 1983). The online movement corrections would notably rely on the posterior parietal cortex (Desmurget et al. 1999; Reichenbach et al. 2014) and would be based on the computed difference between the motor goal and the current position of the hand during the movement.

A critical issue in the field of motor control is to understand the link between feedforward and feedback control. This relationship has essentially been tackled by investigating how both types of control respond to perturbations generated in the same domain, either mechanical or visual. In the mechanical domain, largely associated with upper limb proprioception, several studies demonstrated that online responses to mechanical perturbations applied to the moving arm are adapted to the force field in which the movement evolves (Cluff and Scott 2013; Crevecoeur and Scott 2013; Kimura and Gomi 2009; Maeda et al. 2018; Wagner and Smith 2008; Wang et al. 2001). For instance, Wagner and Smith (2008) showed that after learning to move the arm in a velocity-dependent force field, the motor response to force pulse applied on the arm is immediately scaled to the altered force field. More recent findings (Maeda et al. 2018) revealed that when participants learn new intersegmental dynamics involving decreased shoulder muscle activity, the muscle response to unpredictable mechanical perturbations is also tuned to the adapted feedforward control. In the visual domain, adaptation to visual feedback rotation was shown to affect visually-based movement corrections. For instance, responses to sudden visual shifts of hand or target positions were found to be perfectly 
scaled to the level of adaptation of feedforward control (Dvorkin et al. 2009; Hayashi et al. 2016; Saijo and Gomi 2010; Telgen et al. 2014).

In the above studies, the perturbations of the feedforward and feedback control were likely encoded in a common coordinate system because they both pertain to the same domain (i.e., intrinsic and extrinsic systems for the mechanical and visual perturbations respectively, Krakauer et al. 1999). This sensory context most likely reduced the complexity of the sensorimotor processes involved in online movement corrections. This hypothesis is consistent with the observations made in several studies that the integration of sensorimotor information in a same coordinate system leads to smaller noise and bias than when different coordinate systems are involved (Manson et al. 2019; Sarlegna et al. 2009; Tagliabue and McIntyre 2011; Tong et al. 2002). As a result, the use of a common sensory modality for encoding target position and controlling hand trajectory might induce smaller endpoint error and shorter correction latencies when the motor goal suddenly changes during reaching movements (Manson et al. 2019; Reichenbach et al. 2009). Then it follows that movement corrections could be impaired when the feedforward and feedback control involve different coordinate systems, particularly when the time for implementing these corrections is reduced as is the case with rapid movements.

The question raised, therefore, is whether the strong link between feedforward and feedback control revealed in studies on goal-directed arm movements holds when both the sustained and the unpredictable perturbations pertain to different domains. Diamond et al. (2015) addressed a similar question by assessing the changes of grip and load forces produced by subjects transporting a hand-held object whose dynamics varied according to its position in space. After adaptation to the new object dynamics, the visually-indicated location where the subjects had to bring the object occasionally changed position during the arm movements. The authors found that the corrections of the load and grip forces were perfectly tuned to the change 
118 of the object's dynamics caused by the new path taken by the hand. They concluded that the

119 internal models of novel object dynamics were integrated into visually-driven corrective arm

120 movements (Diamond et al. 2015). However, the spatiotemporal characteristics of online

121 corrections of the arm trajectory were not addressed in this study. Therefore, while their results

122 are consistent with a close link between feedforward and feedback control, several key

123 questions remained unanswered regarding the online control of arm movement when the

124 perturbations of feedforward and feedback control relate to different domains. Foremost among

125 these, it remains unclear if the feedback control was readily optimized from the very first time

126 subjects had to reorient their movements according to the new target position. Moreover, being

127 a critical function of the feedback control system, the reorganization of the muscular activity

128 during perturbation trials was not considered in Diamond et al.'s (2015) study.

129 In the present study, we specifically addressed these issues by analyzing the

130 spatiotemporal dynamics of the arm movements and arm muscle electromyography when

131 participants corrected their hand trajectories in response to a sudden change of target position

132 (visual domain) after adaptation of the feedforward control to an altered gravitoinertial force

133 field (mechanical domain). 


\section{Materials and Methods}

135 Participants

136

137

138

Sixteen right-handed participants (mean age $=22.8 \pm 2.5$ years, 7 females), all naïve to the goal of the experiment, participated after giving their informed consent. None reported a sensorimotor deficit and all had normal or corrected-to-normal vision. The study was approved by the local ethical committee of the Institute of Movement Sciences and was performed in accordance with the standards of the Declaration of Helsinki.

\section{Experimental setup}

The experiment was carried out in a dark room. Participants were seated in a bucket seat placed at the center of a motorized rotating platform. A headrest kept their head immobile. In front of them, a horizontal board was positioned $45 \mathrm{~cm}$ above the seat. A micro-switch located $25 \mathrm{~cm}$ in front of the participants' mid-trunk, was used to standardize the initial position of the reaching index finger. Two visual targets (red light-emitting diodes) were located along the midline body axis at a distance of respectively $25 \mathrm{~cm}$ (Tclose) and $35 \mathrm{~cm}$ (Tfar) from the microswitch (see Fig. 1). Target lighting was controlled by homemade software (Docometre ${ }^{\circledR}$ ). The 3D index finger position was recorded at $200 \mathrm{~Hz}$ with an optical motion capture system (Codamotion CXS and ActiveHub; Charnwood Dynamics, Leicestershire, UK) that tracked the position of an infrared active marker fixed to the tip of the right index finger.

Insert Fig. 1 about here

Surface electromyographic activity (EMG) of arm muscles was analyzed to assess changes in motor commands in response to a target jump in an altered gravitoinertial force field (BIOPAC Systems, Inc., Santa Barbara, CA, 1000 Hz). We recorded the activity of two agonist 
158

muscles (clavicular head of pectoralis; short head of triceps brachii) and two antagonist muscles

159 (posterior deltoid; lateral head of biceps brachii) involved in the reaching movements. Torques

160

161

162

163

164

165

166

167 Germany).

168

169

170

171

172 release of the micro-switch. In $20 \%$ of the trials (pseudo-randomly distributed), a target jump

173 from Tclose to Tfar (Tjump condition) occurred on release of the micro-switch, with Tfar target

174 remaining lit for $100 \mathrm{~ms}$. Under these conditions, movement corrections are deemed to be

175 visually-based, even if visual information was withheld during the reaches (see Brouwer and

176 Knill 2007). For all conditions, participants were instructed to reach towards the target in a

177 single movement and to avoid corrective movements after their index finger touched the board

178 (i.e., considered here as offline corrections). An auditory cue provided $1.6 \mathrm{~s}$ after movement

179 onset informed participants to slowly return their forefinger to the starting position.

180

The experimental session consisted of three successive phases (see Fig. 1): 

towards Tclose and Tfar (5 trials for each randomly-presented target). They were explicitly

183 informed that there would be no target jump in this set of trials. Then, participants performed 50 reaches towards Tclose and Tjump (40 Tclose and 10 Tjump randomly presented). Before starting this last set of trials, the participants were informed that a change of target position could occur after movement onset.

PER phase (platform rotation): While the participants had their index finger on the starting position, the velocity of the rotating platform was brought to a 120 deg. $\mathrm{s}^{-1}$ plateau in 80 s. The counterclockwise rotation generated both Coriolis and centrifugal forces on the moving $\mathrm{arm}^{1}$. According to the laws of physics, the Coriolis force was orthogonal to the movement path and clockwise (i.e., opposite to the direction of platform rotation). The centrifugal force was in the direction of the movement path. The series of trials only started $30 \mathrm{~s}$ after the platform reached a constant velocity, i.e. when body rotation was no longer perceived (Benson, 1990; George et al. 2011). The participants were instructed to remain still until the start of the first trial. An infrared camera was used to verify their compliance with this instruction. Then, participants performed 30 reaches towards Tclose and Tfar (15 trials for each randomlypresented target: PER-initial phase), a number of trials that has been found sufficient to adapt feedforward control to Coriolis and centrifugal forces through proprioceptive feedback control (Coello et al. 1996; Franklin et al. 2007; Lackner and Dizio 1998). After this set of trials, participants performed 50 reaches towards Tclose and Tjump (40 Tclose and 10 Tjump randomly presented: PER-final phase). As in the PRE phase, before both sets of trials participants were told whether or not target position could change at movement onset.

POST phase (no rotation): At the end of the PER phase, the participants remained still with their forefinger on the starting position until an $80 \mathrm{~s}$ deceleration brought the platform to complete immobilization. For the reason explained regarding the PER phase, the first trial of 
the POST phase only started $30 \mathrm{~s}$ after the platform became stationary. Participants performed 6 reaches towards Tclose and Tfar (3 trials for each randomly-presented target: POST-initial phase), followed by 25 reaches towards Tclose and Tjump (20 Tclose and 5 Tjump randomly presented: POST-final phase). Again, participants were told before both sets of trials whether or not target position could change at movement onset. performing 6 reaching movements towards Tclose and Tfar (3 trials for each randomly213 presented target) and 15 reaching movements towards Tclose and Tjump (12 Tclose and 3 214 Tjump randomly presented) in a non-altered gravitoinertial force field.

\section{$215 \quad$ Kinematic analyses}

Data were analyzed using Matlab (Mathworks, Natick, MA, USA). Raw positional data

217 of the marker located on the reaching index finger were low-pass filtered with a dual-pass 218 Butterworth (cut-off frequency: $10 \mathrm{~Hz}$; order: 3). To determine whether participants actually 219 adapted to the altered gravitoinertial force field, we first compared reaching performance in the 220 single-step trials (i.e., Tclose and Tfar) from each experimental phase. Following common 221 procedure for sensorimotor adaptation studies (see Lackner and Dizio 1994; Sarlegna et al. 222 2010), analyses for each variable of interest (see below) included the mean computed from all 223 trials of the PRE phase with no target jump possible (baseline), the first and last trials of the 224 PER phase, and the first and last trials of the POST phase. Note that no target jump was possible 225 in these PER and POST trials.

Several variables were computed to evaluate movement performance. Tangential peak 227 velocity (PV) of the index finger movement was calculated from the marker $\mathrm{x}$ and $\mathrm{y}$ coordinates. 228 Movement time (MT) was calculated as the time between movement onset and offset, identified 229 as when tangential velocity exceeded and fell below $2 \%$ of PV, respectively. 
We computed the lateral endpoint error corresponding to the signed deviation of the

231 finger at movement offset relative to the target on the x-axis. This mediolateral axis represented

232 the main direction of the rotation-induced Coriolis force on the reaching arm. Negative and

233 positive lateral endpoint errors indicated leftward and rightward finger deviations with respect

234 to the target, respectively. Longitudinal endpoint error corresponded to the signed final

235 deviation relative to the target on the y-axis. This anteroposterior axis represented the main

236 direction of centrifugal forces. Negative and positive longitudinal endpoint errors indicated

237 target undershoot and overshoot, respectively. For both Tclose and Tfar, endpoint errors were

238 rebased relative to the mean endpoint positions computed from the first 10 trials of the PRE

239 phase. For these trials, the participants knew that the targets would remain stationary. Finally,

240 we measured the angle between the vector connecting starting position and target, and the

241 vector connecting starting position and finger position to identify the maximum finger angular

242 deviation from movement onset to time to PV. Because of sensorimotor delays, feedback

243 control has little influence on movement before PV (Komilis et al. 1993), variables measured

244 before this kinematics landmark are considered as resulting mainly from feedforward control.

The second step consisted in comparing the Tjump trials from the different phases. We excluded trials exhibiting offline corrective movements, defined as those where, between movement onset and movement offset, tangential velocity was $0 \mathrm{~cm} \cdot \mathrm{s}^{-1}$ or the $\mathrm{z}$ coordinate of 248 the index finger equaled the $\mathrm{z}$ coordinate of the targets' surface (see Fig. 2C). The remaining 84\% of total Tjump trials (with no significant difference in proportion across phases) were examined to identify whether or not they contained overt online secondary corrections (Fig. 2A and 2B, respectively). Trials were considered as involving such secondary corrections when

252 they exhibited a velocity bounce following a first deceleration phase (i.e., after PV, see 253 Boulinguez et al. 2001). Because secondary corrections are deemed to be under online control, 254 only Tjump trials with these observable corrections were kept (82\% of the Tjump trials showed 
255

secondary corrections with no significant difference in proportion across phases). Note that trials without such online corrections showed large longitudinal endpoint errors (on average, $5.17 \mathrm{~cm}$ undershoot). This observation attests that the secondary corrections, as identified using the criteria described above, helped preserving movement accuracy. Since 4 participants did not satisfy the double inclusion criteria (i.e., absence of offline correction and presence of overt online correction) in at least one phase, the results of 12 out of 16 participants were kept for this second step of data analyses.

Insert Fig. 2 about here

$\mathrm{cm} . \mathrm{s}^{-2}$ after the first acceleration and deceleration phases. From this time, we computed the secondary correction time (time between movement onset and beginning of secondary correction), the PV of the secondary correction (maximum tangential velocity between beginning of secondary correction and movement offset), and relative time to PV of the secondary correction (TPV), computed in percentage relative to the total duration of the secondary correction (from the secondary correction time to the movement offset; see Fig. 2A). For each of these variables, we compared the mean computed from all Tjump trials of the PRE jump phase, the first and the last Tjump trial of the PER jump phase and the first and the last Tjump trial of the POST jump phase. Note that splitting the data into different phases (i.e., PERinitial PER- final and POST-initial POST-final) allowed us to determine 1) if the online correction observed during the first Tjump after adaptation or re-adaptation was functional and 2) if the online correction in the PER jump phase improved after practice (PER-initial vs PERfinal) as is the case during sensorimotor adaptation and as revealed in the PER phase (without target jump) of the present study. The exclusion of some trials due to the criteria used to identify 
280

281

282

283

284

285

286

287

288

289

290

291

292

293

294

295

296

297

298

299

movement correction meant that the Tjump trials analyzed were not always the first or the last

Tjump trial; however, they fell mainly within the first (88\%) or last (92\%) two trials of the PER jump and POST jump phases.

\section{EMG analyses}

Raw EMG data were filtered with a Butterworth type band-pass filter (cut-off frequency: 20-400 Hz; order: 4) to minimize signals unrelated to the physiological frequency of muscle activity (van Boxtel 2001). After centering around the mean and rectifying the signal, a low-pass Butterworth filter was applied twice (forward and backward to remove phase shift) with a $3 \mathrm{~Hz}$ cut-off frequency (order: 3 ) to create an envelope of the EMG signal. The activity of each muscle was normalized and expressed as a percentage of their maximum activity observed during the Tjump trials in the PRE phase.

EMG analyses were performed on the Tjump trials of 12 participants (selection procedure described above). For each muscle (pectoralis, biceps, posterior deltoid, triceps) and each phase (PRE jump, PER-initial jump, PER-final jump, POST-initial jump, POST-final jump), activation level was estimated by computing the EMG Root Mean Square (RMS). For each trial, EMG RMS calculation started 90 ms before the secondary correction (to allow for 'motor time'; Soechting and Lacquaniti 1983) and ended at the PV secondary correction. Computed over this time window, the EMG RMS can be considered to provide a good estimation of the motor command during the secondary corrections.

\section{Statistical analyses}

To determine whether participants adapted to the altered gravitoinertial force field before the first Tjump trial, for each movement we compared the kinematics variables computed for the different phases without Tjump trials (PRE, PER-initial, PER-final, POST-initial, POST-final) using repeated measures ANOVAs. To investigate online corrections following 
304

305

displacements of the visual target, we compared the kinematics and EMG variables computed for the different phases of the Tjump trials (PRE jump, PER-initial jump, PER-final jump, POST-initial jump, POST-final jump) using repeated measures ANOVAs.

All statistical analyses were performed with Statistica software (StatSoft, Inc., OK, USA). The normal distribution of data for each variable was confirmed by KolmogorovSmirnov tests. Post-hoc analyses were carried out using Newman-Keuls tests. Significance threshold was set at $\mathrm{p}<0.05$ for all analyses.

\section{Results}

\section{Confirmation of feedforward control adaptation}

The reaching trajectories recorded in the different phases prior to the Tjump trials indicated that participants adapted to the altered gravitoinertial force field (Fig. 3). More specifically, movements performed before the force field change (PRE phase) showed nearly rectilinear trajectories and final endpoint positions close to the target. However, in the first trial performed in the modified force field (PER-initial trial), the subject's reaching finger deviated to the right and overshot the target, presumably due to Coriolis and centrifugal forces, respectively. After several trials in the altered gravitoinertial force field (PER-final trial), movements became straighter and more accurate. In contrast, the first movements performed after the gravitoinertial force field returned to normal (POST-initial) deviated widely to the left of the target. Finally, at the end of the POST phase (POST-final), reaching movements were almost as rectilinear and as accurate as during the PRE phase.

Insert Fig. 3 about here 
The statistical analyses performed on the different kinematics variables revealed how the exposure phases affected reaching movements. The ANOVA performed on lateral endpoint errors showed a significant phase effect $\left(F_{(4,56)}=21.33\right.$; $p<0.001$; Fig. 4A). Post-hoc analysis showed that reaching movements performed during PER-initial and POST-initial phases were respectively more deviated to the right and to the left of the target than those performed in the other phases. Consistent with the adaptation of the feedforward control to the altered force field, the lateral endpoint errors did not significantly differ between PRE, PER-final and POST-final phases.

The ANOVA also revealed a significant phase effect on longitudinal endpoint errors $\left(\mathrm{F}_{(4,56)}=7.25 ; \mathrm{p}<0.001\right.$; Fig. 4B). Post-hoc analyses revealed that reaching movements performed in the PER-initial phase overshot the target and had greater amplitudes than in all other phases. While the longitudinal endpoint errors did not significantly differ between the PRE and the POST-initial phases, movements performed during the POST-initial phase had smaller amplitude than during the PER-final and POST-initial phases. Together, these results denote some signs of feedforward control adaptation of movement extent in the new force field.

Maximum finger angular deviation before PV was also impacted by phase $\left(\mathrm{F}_{(4,56)}=\right.$ 12.43; $\mathrm{p}<0.001$; Fig. 5). Post-hoc analyses showed that the reaching movements performed in the PER-initial and POST-initial phases were significantly more deviated to the right and to the 348 left than movements in the PRE and PER-final phases, respectively. On the other hand, before PV, movements in the POST-initial phase was more deviated to the left than those in the PRE phase $(\mathrm{p}<0.001)$. Kinematic landmarks falling before peak velocity are considered to mainly 
352 feedforward control was adapted to the altered gravitoinertial force field, consistent with

353 findings from previous studies that used similar types of paradigms (Lackner and Dizio 1994;

354 Sarlegna et al. 2010).

355

356

357

358

359

360

361

362

363

364

365

366

367

368

369

370

371

372

373

374

Insert Fig. 5 about here

\section{Comparison of Tfar and Tjump trials}

The presence of a target jump during reaching fundamentally changed the spatiotemporal organization of the movement. This can be seen in Fig. 6, which shows the respective mean tangential velocities of all Tfar and valid Tjump trials, where the same spatial goal had to be reached with or without a change of target position. Compared to Tfar trials, Tjump trials had a smaller peak velocity and showed a secondary PV after a first deceleration phase. Movement times were also longer in Tjump trials than in Tfar trials (mean: 490 ms \pm 63 ms vs $336 \mathrm{~ms} \pm 56 \mathrm{~ms}$ respectively), as confirmed by a paired t-test $\left(\mathrm{t}_{(11)}=9.73 ; \mathrm{p}<0.001\right)$. These modified kinematics show that visually extracted information on the new target position was integrated into the control of the ongoing reaching movement. Moreover, for Tjump trials, the secondary correction time was much shorter than the reaction time of the primary movement (mean: $278 \mathrm{~ms} \pm 53 \mathrm{~ms}$ vs $411 \mathrm{~ms} \pm 58$ ms respectively; $\mathrm{t}_{(11)}=6.82 ; \mathrm{p}<0.0001$ ). This result concurs with the findings from several studies using double step reaching paradigm which showed that the modification of motor commands under feedback control is faster than the time necessary to produce new motor commands under feedforward control (Day and Lyon 2000; Fautrelle et al. 2010; Kadota and Gomi 2010; Prablanc and Martin 1992; Reichenbach et al. 2009; Saunders and Knill 2003; Smeets et al. 2016). 
Comparison of Tjump trials from the different jump phases significantly between the different experimental phases (PRE jump, PER-initial jump, PERfinal jump, POST-initial jump, POST-final jump). ANOVAs did not reveal significant phase effects on mean $\mathrm{MT}\left(\mathrm{F}_{(4,44)}=1.16 ; \mathrm{p}=0.34\right.$, overall mean $\left.(\bar{x}): 489 \mathrm{~ms} \pm 54 \mathrm{~ms}\right)$, lateral endpoint errors $\left(F_{(4,44)}=1.19 ; \mathrm{p}=0.33, \bar{x}: 0.60 \mathrm{~cm} \pm 0.81 \mathrm{~cm}\right.$; Fig. $\left.7 \mathrm{~A}\right)$ or longitudinal endpoint errors $\left(\mathrm{F}_{(4,44)}=2.19 ; \mathrm{p}=0.09, \bar{x}: 0.85 \mathrm{~cm} \pm 3.21 \mathrm{~cm}\right.$; Fig. 7B $), \mathrm{PV}$ secondary correction $\left(\mathrm{F}_{(4,44)}=\right.$ 1.36; $\mathrm{p}=0.26 ; \bar{x}: 97 \mathrm{~cm} . \mathrm{s}^{-1} \pm 45 \mathrm{~cm} . \mathrm{s}^{-1}$; Fig. 7C), TPV secondary correction $\left(\mathrm{F}_{(4,44)}=0.97 ; \mathrm{p}=\right.$

$0.43 ; \bar{x}: 35 \% \pm 11 \%$; Fig. 7D), and secondary correction time $\left(\mathrm{F}_{(4,44)}=0.88 ; \mathrm{p}=0.48 ; \bar{x}: 278\right.$ $\mathrm{ms} \pm 53 \mathrm{~ms})$

However, the EMG RMS analyses showed that muscle activities recorded during movement corrections differed between phases (see Fig. 8 for comparison between PRE jump and PER-initial jump phases). Notably, the ANOVA revealed a significant phase effect on the EMG RMS for the biceps brachii $\left(\mathrm{F}_{(4,44)}=7.4 ; \mathrm{p}<0.001\right.$; Fig. 9A). Post-hoc analysis showed higher EMG RMS values in the PER-initial jump and PER-final jump phases than in the other 
$401<0.01$; Fig. 9B) and posterior deltoid $\left(\mathrm{F}_{(4,44)}=3.5\right.$; $\mathrm{p}<0.05$; Fig.9C) EMG RMS. For the 402 posterior deltoid, EMG RMS was greater in the PER-initial jump phase than in POST-initial 403 jump and POST-final jump phases. For the pectoralis, EMG RMS was greater in the PER-initial 404 jump phase than in all the other phases.

405

406

Insert Fig. 8 about here

407

408

Finally, the ANOVA performed on the EMG RMS of triceps brachii $\left(\mathrm{F}_{(4.44)}=1.7 ; \mathrm{p}=\right.$ 409 0.16; Fig. 9D) did not show a significant phase effect.

410 
414

415

416

417

418

419

420

421

423

424

425

426

428

430

431

433

434

435

\section{Discussion}

The present study explored the link between feedback and feedforward control by looking at whether, when motor commands are adapted to a new force field, online control of arm movement remains effective under a perturbation of a different nature. In a two-step experimental protocol, participants first adapted feedforward control to the mechanical perturbation of an altered gravitoinertial force field by reaching towards visual targets while seated in a rotating environment. Then, we assessed whether online control mechanisms were readily functional in this altered force field by examining the participants' arm responses to unpredictable changes in target position (i.e., visual perturbation) at movement onset. Together, kinematics and EMG analyses showed for the first time that although feedforward and feedback control were driven by different sensory inputs, feedback control was remarkably functional, from the very first time participants encountered a target jump in the altered force field.

\section{Validation of adaptation to an altered gravitoinertial force field}

The first reaching movement performed by the participants after being re-exposed to a normal gravitoinertial force field (i.e., in POST-initial phase) showed wide trajectory deviation. The deviation was in the opposite direction to the Coriolis force exerted on the arm during the preceding series of reaches in the altered force field. This so-called post-effect confirmed that the feedforward control responsible for triggering the movements was adapted to the force field change induced by platform rotation (Coello et al. 1996; Franklin et al. 2007; Lackner and Dizio 1998). According to prevailing theories of motor control, this adaptation reflects the updating of internal models of reaching, based on the new environment dynamics detected through proprioceptive information processing (Wolpert et al. 2011).

Adaptation to externally-induced centrifugal forces has received little attention in previous studies. Those using a paradigm in which participants were seated on-axis of a rotating 
438 platform (Coello et al. 1996; Lackner and Dizio 1994), or off-axis (Bourdin et al. 2001; Kurtzer 439 et al. 2005; Lackner and Dizio 1998), showed that centrifugal force had no significant effects 440 on movement accuracy, and that exposure to this force did not lead to post-effects. On the 441 contrary, our participants widely overshot the target during their first reach under externally442 induced centrifugal force. The smaller longitudinal errors reported in previous studies may stem 443 from the fact that, prior to reaching, the hand position appeared to be farther from the rotation 444 axis, even when participants were seated above this axis (see Coello et al. 1996; Lackner and 445 Dizio 1994). This gave participants the opportunity to perceive the centrifugal force applied to their body before reaching, and to take it into account when planning their movements. Similar 447 integration of the gravitoinertial context prior to movement execution has being reported in 448 several studies (Blouin et al. 2015; Bockisch and Haslwanter 2007; Cohn et al. 2000; Macaluso 449 et al. 2017). In our study, however, before initiating their movements, participants' hands were 450 positioned very close to the rotation axis, a position that prevented detection of the centrifugal force and anticipation of its effects on the arm during the movement. performed in the altered gravitoinertial force field, the post-effect observed for this variable greatly differed from that revealed for the directional errors. Indeed, the participants' longitudinal errors when first re-exposed to a normal force field did not significantly differ from those produced prior to the force field alteration (PRE phase). However, the amplitude of the first post-rotation movement was significantly smaller than the last movement performed during the rotation (PER-final) and the last movement performed after being re-exposed to a normal force field (POST-final). This pattern of results suggests that participants had begun to 460 adapt their movement amplitude by the end of exposure to the altered gravitoinertial force field, 461 but to a lesser extent compared to the adaptation shown for movement direction (assessed here 462 using lateral endpoint errors). 
Motor responses to target jump in altered force field

463

464

465

466

467

468

469

470

471

472

473

474

475

476

477

478

479

480

481

482

483

484

485

486

487

These differing capacities to adapt movement amplitude and direction could be explained with reference to the vectorial coding model of movements. According to this model, motor commands are planned according to the direction and the amplitude of a hand-target vector computed by the brain (Davare et al. 2012; Favilla et al. 1990; Krakauer et al. 1999, 2000; Messier and Kalaska 2000; Rossetti et al. 1995). Importantly, the fact that movement direction has to be specified before movement onset (Fleury et al. 1994; Ghez et al. 1989; Paulignan et al. 1991; van Sonderen et al. 1988), unlike movement amplitude (Favilla et al. 1990; Ghez et al. 1989; Sarlegna et al. 2010) might place greater stress on planning movement direction than movement amplitude. This could be responsible for the observation made here and in previous studies (Bourdin et al. 2001; Coello et al. 1996; Kurtzer et al. 2005; Lackner and Dizio 1994, 1998) that adaptation to Coriolis force is faster than adaptation to centrifugal force.

\section{The strong relationship between feedforward and feedback control is not context-dependent}

To our knowledge, the link between feedforward and feedback control in different domains has only been assessed by Diamond et al. (2015). In their study, the feedforward control was first adapted by having participants transport several times a hand-held object whose load force depended on its position within the working space. Then, the experimenter changed the (visual) location where the participants had to bring the object while they were moving it. The authors showed that participants could remarkably scale grip and load forces according to the change of object load force associated with the movement correction. However, no analyses related to reaching corrections were reported. The authors simply indicated that the reaching errors were greater than $0.5 \mathrm{~cm}$ in only $17 \%$ of the trials but without specifying whether these trials were gathered within the first attempts to reach the new target location. In the present study, the thorough investigation of movement corrections revealed that the spatiotemporal characteristics of the corrective movements were strikingly similar between 
normal and altered gravitoinertial force fields. This strong similarity was observed even when participants experienced their first target jump in the altered gravitoinertial force field (PERinitial jump phase), and for all movement parameters (e.g., lateral and longitudinal endpoint errors, movement duration, correction latency). Even the fine kinematics variables, such as the peak velocity of the corrective movement and its relative time of occurrence, were not impacted by the change in gravitoinertial forces. The remarkable spatiotemporal stability of online corrective responses, including during the first experience of a target jump in the new force field, supports the hypothesis that the adaptation of feedforward control readily transferred to feedback control.

Because rotating the environment in which individuals moved their arm created Coriolis and centrifugal forces, similar movement corrections could be expected to require different muscle torques in normal and altered gravitoinertial force fields. This was confirmed by our EMG analyses, which showed greater activity of the biceps (PER-initial jump and PER-final jump phases), pectoralis (PER-initial jump phase) and posterior deltoid (PER-initial jump compared to POST-initial jump and POST-final jump phases) muscles during the movement corrections observed in the altered gravitoinertial force field.

When participants reached towards the targets while being rotated in the counterclockwise direction, Coriolis force pushed the arm to the right. The increased activities of the right biceps (elbow flexor) and pectoralis (arm adductor) muscles may therefore have helped to offset Coriolis force and maintain a rectilinear hand trajectory during movement corrections. On the other hand, because it was in the same direction as the movement trajectory, centrifugal force facilitated reaching movements during platform rotation. Thus, the increased activation of the biceps and posterior deltoid muscles may also have slowed down the hand being pushed by centrifugal force as it moved away from the center of rotation. Importantly, this fine-tuning of biceps and pectoralis muscle activities was also effective from the first time 
513 the target changed its position during the reaching movements. These results, which corroborate

514 the kinematic analyses, suggest readily functional online control when feedforward control is 515 adapted to new gravitoinertial constraints.

Increasing muscle activity when learning new arm dynamics is known to reduce movement errors and to accelerate the adaptation process (Heald et al. 2018). This raises the possibility that the greater activity observed here in the pectoralis, biceps brachii and posterior deltoid muscles during movement corrections may have improved the efficiency of feedback 520 control. This hypothesis requires further testing, but it is in line with the suggestion that 521 increasing the activity of arm muscles enhances visuomotor feedback gain and improves arm responses to sudden and unpredictable visual perturbations (Franklin et al. 2012). Because pectoralis muscle was less active on the last Tjump trial, co-contraction or muscle stiffness may not be the motor strategies developed by the brain to counter the forces, at least in the longer term. The decrease of pectoralis muscle activity observed here over time could be explained by the optimal control theory (Diedrichsen et al. 2010; Todorov 2004). An optimization of motor command over Tjump trials may be based on a reorganization of muscular synergies (d'Avella et al. 2006) to minimize energy cost while maintaining spatial accuracy. The fact that pectoralis muscle was the main agonist muscle in the present reaching task, and therefore the most energy costing, could explain why the diminution of EMG activity was only effective in this muscle. essentially been investigated by testing both types of control under perturbations pertaining to 533 a common domain, e.g., mechanical or visual. Thus, these studies found feedforward and 534 feedback control to be driven either by proprioceptive (Cluff and Scott 2013; Crevecoeur and 535 Scott 2013; Kimura and Gomi 2009; Maeda et al. 2018; Wagner and Smith 2008; Wang et al. 536 2001) or by visual (Dvorkin et al. 2009; Hayashi et al. 2016; Saijo and Gomi 2010; Telgen et 537 al. 2014) feedback. They showed effective corrections from the first movement perturbation, 
538 confirming that in this context, feedforward and feedback controls are closely linked (Telgen

539 et al. 2014; Wagner and Smith 2008). The present study demonstrates that the changes resulting

540 from feedforward control adaptation are readily available to feedback-based processes in

541 contexts where the two modes of control are facing different types of perturbation. While force

542 field and visuomotor adaptations have been found to involve distinct neural networks (Donchin

543 et al. 2012; Rabe et al. 2009), our findings suggest that these networks are functionally (directly

544 or indirectly) interconnected, thereby allowing greater flexibility in the control of arm 545 movements.

546 In conclusion, we demonstrated that after adapting feedforward control to the 547 mechanical perturbation of a sustained altered gravitoinertial force field, the internal model 548 based on arm dynamics and environmental properties led to functional feedback control driven 549 by visual information about the new target position. Thus, when feedforward control provides 550 a state estimate of arm dynamics under mechanical perturbation, feedback control processes 551 may be able to use visual information to produce adapted motor commands that also take into 552 account the mechanical changes and their consequences on the upper limb. 


\section{Acknowledgments}

555 The authors wish to thank F. Buloup for technical support and assistance with

556 experiments. The authors are also grateful to the participants in this study.

\section{Grants}

558

This work was supported by APR Grants (“FLEXMOVE” Grants 884 \& 944) from the

559 French National Space Research Centre (CNES), and grants from the French Research Council

560 (ANR Motion - 14-CE30-0007).

\section{Disclosures}

562 The authors declare that the research was conducted in the absence of any commercial or

563 financial relationships that could be construed as a potential conflict of interest.

\section{Author contributions}

565 LC designed and performed the experiment, analyzed data and wrote the paper; LB and

566 JB designed the experiment and wrote the paper. 


\section{References}

569 d'Avella A, Portone A, Fernandez L, Lacquaniti F. Control of Fast-Reaching Movements by Muscle Synergy Combinations. J Neurosci 26: 7791-7810, 2006.

Benson AJ. Sensory Functions and Limitations of the Vestibular System. In: The Perception and Control of Self-Motion, edited by Warren R. \& Wertheim AH. Hillsdale, NJ: Lawrence Erlbaum Associates, Inc., 1990, p. 145-170.

Blouin J, Bresciani J-P, Guillaud E, Simoneau M. Prediction in the vestibular control of arm movements. Multisensory Res 28: 487-505, 2015.

Bockisch CJ, Haslwanter T. Vestibular contribution to the planning of reach trajectories. Exp Brain Res 182: 387-397, 2007.

Boulinguez $\mathbf{P}$, Nougier V, Velay JL. Manual asymmetries in reaching movement control. I: Study of right-handers. Cortex 37: 101-122, 2001.

580 Bourdin C, Gauthier G, Blouin J, Vercher JL. Visual feedback of the moving arm allows 581 complete adaptation of pointing movements to centrifugal and Coriolis forces in human 582 subjects. Neurosci Lett 301: 25-28, 2001.

583 van Boxtel A. Optimal signal bandwidth for the recording of surface EMG activity of facial, jaw, oral, and neck muscles. Psychophysiology 38: 22-34, 2001.

Brindle TJ, Nitz AJ, Uhl TL, Kifer E, Shapiro R. Kinematic and EMG characteristics of simple shoulder movements with proprioception and visual feedback. J Electromyogr Kinesiol 16: 236-249, 2006.

Brouwer A-M, Knill DC. The role of memory in visually guided reaching. $J$ Vis 7: 6.1-12, 2007.

590 Churchland MM, Santhanam G, Shenoy KV. Preparatory activity in premotor and motor cortex reflects the speed of the upcoming reach. J Neurophysiol 96: 3130-3146, 2006.

Cluff T, Scott SH. Rapid feedback responses correlate with reach adaptation and properties of novel upper limb loads. J Neurosci 33: 15903-15914, 2013.

Coello Y, Orliaguet JP, Prablanc C. Pointing movement in an artificial perturbing inertial field: a prospective paradigm for motor control study. Neuropsychologia 34: 879-892, 1996.

Cohn JV, DiZio P, Lackner JR. Reaching during virtual rotation: context specific compensations for expected coriolis forces. J Neurophysiol 83: 3230-3240, 2000.

Crevecoeur F, Scott SH. Priors engaged in long-latency responses to mechanical perturbations suggest a rapid update in state estimation. PLoS Comput Biol 9: e1003177, 2013.

600 Davare M, Zénon A, Pourtois G, Desmurget M, Olivier E. Role of the medial part of the 601 intraparietal sulcus in implementing movement direction. Cereb Cortex 22: 1382-1394, 2012.

602 Day BL, Lyon IN. Voluntary modification of automatic arm movements evoked by motion of 603 a visual target. Exp Brain Res 130: 159-168, 2000. 
604 Desmurget M, Epstein CM, Turner RS, Prablanc C, Alexander GE, Grafton ST. Role of 605 the posterior parietal cortex in updating reaching movements to a visual target. Nat Neurosci 2: 606 563-567, 1999.

607 Desmurget M, Grafton ST. Forward modeling allows feedback control for fast reaching 608 movements. Trends Cogn Sci 4: 423-431, 2000.

609 Diamond JS, Nashed JY, Johansson RS, Wolpert DM, Flanagan JR. Rapid Visuomotor 610 Corrective Responses during Transport of Hand-Held Objects Incorporate Novel Object 611 Dynamics. J Neurosci Off J Soc Neurosci 35: 10572-10580, 2015.

612 Diedrichsen J, Shadmehr R, Ivry RB. The coordination of movement: optimal feedback 613 control and beyond. Trends Cogn Sci 14: 31-39, 2010.

614 Donchin O, Rabe K, Diedrichsen J, Lally N, Schoch B, Gizewski ER, Timmann D. 615 Cerebellar regions involved in adaptation to force field and visuomotor perturbation. $J$ $616 \quad$ Neurophysiol 107: 134-147, 2012.

617 Dvorkin AY, Kenyon RV, Keshner EA. Effects of roll visual motion on online control of arm 618 movement: reaching within a dynamic virtual environment. Exp Brain Res 193: 95-107, 2009.

619 Fautrelle L, Prablanc C, Berret B, Ballay Y, Bonnetblanc F. Pointing to double-step visual 620 stimuli from a standing position: very short latency (express) corrections are observed in upper 621 and lower limbs and may not require cortical involvement. Neuroscience 169: 697-705, 2010.

622 Favilla M, Gordon J, Hening W, Ghez C. Trajectory control in targeted force impulses. VII. 623 Independent setting of amplitude and direction in response preparation. Exp Brain Res 79: 530624 538, 1990.

625 Fleury M, Bard C, Auddifren M, Teasdale N, Blouin J. The attentional cost of amplitude 626 and directional requirements when pointing to targets. Q J Exp Psychol: Hum Exp Psychol 47A: 627 481-495, 1994.

628 Franklin DW, Liaw G, Milner TE, Osu R, Burdet E, Kawato M. Endpoint stiffness of the 629 arm is directionally tuned to instability in the environment. J Neurosci 27: 7705-7716, 2007.

630 Franklin S, Wolpert DM, Franklin DW. Visuomotor feedback gains upregulate during the 631 learning of novel dynamics. J Neurophysiol 108: 467-478, 2012.

632 Gaveau J, Berret B, Angelaki DE, Papaxanthis C. Direction-dependent arm kinematics 633 reveal optimal integration of gravity cues. eLife 5, 2016.

634 George RJS, Day BL, Fitzpatrick RC. Adaptation of vestibular signals for self-motion 635 perception. J Physiol 589: 843-853, 2011.

636 Ghez C, Hening W, Favilla M. Gradual specification of response amplitude in human tracking 637 performance. Brain Behav Evol 33: 69-74, 1989.

638 Hayashi T, Yokoi A, Hirashima M, Nozaki D. Visuomotor map determines how visually 639 guided reaching movements are corrected within and across trials. eNeuro 3, 2016. 
640 Heald JB, Franklin DW, Wolpert DM. Increasing muscle co-contraction speeds up internal 641 model acquisition during dynamic motor learning. Sci Rep 8: 16355, 2018.

642 Kadota K, Gomi H. Implicit visuomotor processing for quick online reactions is robust against 643 aging. J Neurosci Off J Soc Neurosci 30: 205-209, 2010.

644 Kimura T, Gomi H. Temporal development of anticipatory reflex modulation to dynamical 645 interactions during arm movement. J Neurophysiol 102: 2220-2231, 2009.

646 Komilis E, Pélisson D, Prablanc C. Error processing in pointing at randomly feedback647 induced double-step stimuli. J Mot Behav 25: 299-308, 1993.

648 Krakauer JW, Ghilardi MF, Ghez C. Independent learning of internal models for kinematic 649 and dynamic control of reaching. Nat Neurosci 2: 1026-1031, 1999.

650 Krakauer JW, Pine ZM, Ghilardi MF, Ghez C. Learning of visuomotor transformations for 651 vectorial planning of reaching trajectories. J Neurosci 20: 8916-8924, 2000.

652 Kurtzer I, DiZio PA, Lackner JR. Adaptation to a novel multi-force environment. Exp Brain 653 Res 164: 120-132, 2005.

654 Lackner JR, Dizio P. Rapid adaptation to Coriolis force perturbations of arm trajectory. $J$ 655 Neurophysiol 72: 299-313, 1994.

656 Lackner JR, Dizio P. Gravitoinertial force background level affects adaptation to coriolis force 657 perturbations of reaching movements. J Neurophysiol 80: 546-553, 1998.

658 Macaluso T, Bourdin C, Buloup F, Mille M-L, Sainton P, Sarlegna FR, Vercher J-L, 659 Bringoux L. Sensorimotor reorganizations of arm kinematics and postural strategy for 660 functional whole-body reaching movements in microgravity. Front Physiol 8: 821, 2017.

661 Maeda RS, Cluff T, Gribble PL, Pruszynski JA. Feedforward and feedback control share an 662 internal model of the arm’s dynamics. J Neurosci 38: 10505-10514, 2018.

Manson GA, Blouin J, Kumawat AS, Crainic VA, Tremblay L. Rapid online corrections 664 for upper limb reaches to perturbed somatosensory targets: evidence for non-visual 665 sensorimotor transformation processes. Exp Brain Res 237: 839-853, 2019.

666

667 668

669 670

671 672

673 674
Maschke M, Gomez CM, Ebner TJ, Konczak J. Hereditary cerebellar ataxia progressively impairs force adaptation during goal-directed arm movements. J Neurophysiol 91: 230-238, 2004.

Messier J, Kalaska JF. Covariation of primate dorsal premotor cell activity with direction and amplitude during a memorized-delay reaching task. J Neurophysiol 84: 152-165, 2000.

Mills KR. The basics of electromyography. J Neurol Neurosurg Psychiatry 76 Suppl 2: ii3235, 2005.

Moran DW, Schwartz AB. Motor cortical representation of speed and direction during reaching. J Neurophysiol 82: 2676-2692, 1999. 
675 Papaxanthis C, Pozzo T, McIntyre J. Arm end-point trajectories under normal and micro676 gravity environments. Acta Astronaut 43: 153-161, 1998.

677 Paulignan Y, MacKenzie C, Marteniuk R, Jeannerod M. Selective perturbation of visual 678 input during prehension movements. 1. The effects of changing object position. Exp Brain Res 679 83: 502-512, 1991.

680 Pélisson D, Prablanc C, Goodale MA, Jeannerod M. Visual control of reaching movements 681 without vision of the limb. II. Evidence of fast unconscious processes correcting the trajectory 682 of the hand to the final position of a double-step stimulus. Exp Brain Res 62: 303-311, 1986.

683 Prablanc C, Martin O. Automatic control during hand reaching at undetected two684 dimensional target displacements. J Neurophysiol 67: 455-469, 1992.

685

686

687

688

689

690

691

692

693

694

695

696

697

698

699

700

701

702

703

704

705

706

707

708

709

710

711

Rabe K, Livne O, Gizewski ER, Aurich V, Beck A, Timmann D, Donchin O. Adaptation to visuomotor rotation and force field perturbation is correlated to different brain areas in patients with cerebellar degeneration. J Neurophysiol 101: 1961-1971, 2009.

Reichenbach A, Thielscher A, Peer A, Bülthoff HH, Bresciani J-P. Seeing the hand while reaching speeds up on-line responses to a sudden change in target position. $J$ Physiol 587: 46054616, 2009.

Reichenbach A, Thielscher A, Peer A, Bülthoff HH, Bresciani J-P. A key region in the human parietal cortex for processing proprioceptive hand feedback during reaching movements. NeuroImage 84: 615-625, 2014.

Riehle A, Requin J. Monkey primary motor and premotor cortex: single-cell activity related to prior information about direction and extent of an intended movement. J Neurophysiol 61: 534-549, 1989.

Rossetti Y, Desmurget M, Prablanc C. Vectorial coding of movement: vision, proprioception, or both? J Neurophysiol 74: 457-463, 1995.

Saijo N, Gomi H. Multiple motor learning strategies in visuomotor rotation. PloS One 5: e9399, 2010.

Sarlegna FR, Blouin J. Visual guidance of arm reaching: online adjustments of movement direction are impaired by amplitude control. J Vis 10: 24, 2010.

Sarlegna FR, Blouin J, Bresciani J-P, Bourdin C, Vercher J-L, Gauthier GM. Target and hand position information in the online control of goal-directed arm movements. Exp Brain Res 151: 524-535, 2003.

Sarlegna FR, Malfait N, Bringoux L, Bourdin C, Vercher J-L. Force-field adaptation without proprioception: can vision be used to model limb dynamics? Neuropsychologia 48: 6067, 2010.

Sarlegna FR, Przybyla A, Sainburg RL. The influence of target sensory modality on motor planning may reflect errors in sensori-motor transformations. Neuroscience 164: 597-610, 2009. 
Saunders JA, Knill DC. Humans use continuous visual feedback from the hand to control fast reaching movements. Exp Brain Res 152: 341-352, 2003.

Shadmehr R. Generalization as a behavioral window to the neural mechanisms of learning internal models. Hum Mov Sci 23: 543-568, 2004.

Smeets JB, Oostwoud Wijdenes L, Brenner E. Movement Adjustments Have Short Latencies Because There is No Need to Detect Anything. Motor Control 20: 137-148, 2016.

718 Soechting JF, Lacquaniti F. Modification of trajectory of a pointing movement in response to 719 a change in target location. J Neurophysiol 49: 548-564, 1983.

720 van Sonderen JF, Denier van der Gon JJ, Gielen CC. Conditions determining early 721 modification of motor programmes in response to changes in target location. Exp Brain Res 71: 722 320-328, 1988.

Tagliabue M, McIntyre $\mathbf{J}$. Necessity is the mother of invention: reconstructing missing sensory information in multiple, concurrent reference frames for eye-hand coordination. $J$ Neurosci 31: 1397-1409, 2011.

Telgen S, Parvin D, Diedrichsen J. Mirror reversal and visual rotation are learned and consolidated via separate mechanisms: recalibrating or learning de novo? J Neurosci 34:

\section{13768-13779, 2014.}

Todorov E. Optimality principles in sensorimotor control. Nat Neurosci 7: 907-915, 2004.

730

731

732

733

734

735

736

Tong C, Wolpert DM, Flanagan JR. Kinematics and dynamics are not represented independently in motor working memory: evidence from an interference study. J Neurosci 22: 1108-1113, 2002.

Vindras P, Desmurget M, Prablanc C, Viviani P. Pointing errors reflect biases in the perception of the initial hand position. J Neurophysiol 79: 3290-3294, 1998.

Wagner MJ, Smith MA. Shared internal models for feedforward and feedback control. $J$ Neurosci 28: 10663-10673, 2008.

Wang T, Dordevic GS, Shadmehr R. Learning the dynamics of reaching movements results in the modification of arm impedance and long-latency perturbation responses. Biol Cybern 85: 437-448, 2001.

Wolpert DM, Diedrichsen J, Flanagan JR. Principles of sensorimotor learning. Nat Rev Neurosci 12: 739-751, 2011. 
742

\section{Figure captions}

743

744

745

746

747

748

749

750

751

752

753

754

755

756

757

758

759

760

761

762

763

764

765

766

767

768

769

770

771

772

773

Fig. 1: Experimental setup and temporal organization of the protocol. The first step consisted in validating sensorimotor adaptation using trials without Tjump. For this validation, the statistical analyses were performed using only unperturbed trials of the PRE phase (before rotation), of the PER-initial and PER-final phases (first and last unperturbed trial during rotation), and of the POST-initial and POST-final phases (first and last unperturbed trial after rotation). Note that for all these trials without target jumps, the participants knew that no target jump would occur during their movements. The second step consisted in comparing Tjump trials between the same phases (PRE, PER-initial, PER-final, POST-initial, POST-final). These trials were randomly distributed in a new series of trials including target jump.

Fig. 2: Reaching index finger position in $\mathrm{Z}$ axis (left column) and finger tangential velocity in the sagittal plane (right column) of representative trials showing (A) online secondary correction, (B) absence of online secondary correction and (C) offline secondary correction. Note that $B$ and $C$ types of trials were rejected from the analyses.

Fig. 3: Mean index finger trajectories of all participants (top view) computed in each phase in Tclose trials.

Fig. 4: Means of (A) lateral and (B) longitudinal endpoint errors compared between phases in Tclose and Tfar trials. *: $\mathrm{p}<0.05 ; * *$ : $\mathrm{p}<0.01 ; * * *$ : $<0.001$.

Fig. 5: Maximum angular deviation before PV for the different phases in Tclose and Tfar trials. $*: \mathrm{p}<0.05 ; * *: \mathrm{p}<0.01 ; * * *: \mathrm{p}<0.001$.

Fig. 6: Mean endpoint tangential velocity in the sagittal plane for Tfar (blue line) and Tjump (red line) in all phases. Shaded areas represent positive and negative standard deviations.

Fig. 7: Mean (A) lateral and (B) longitudinal endpoint errors compared between jump phases in Tjump trials. Mean (C) finger peak velocity and (D) relative time to peak velocity during secondary correction compared between phases in Tjump trials. None of these variables were significantly impacted by experimental phases.

Fig. 8: Mean EMG activity of the four recorded muscles for Tjump trials in PRE (blue trace) and PER-initial (red trace) jump phases. The vertical dotted line represents movement onset and the yellow area the time window used to compute EMG RMS during movement corrections.

Fig. 9: Mean EMG RMS of the four recorded muscles computed during the temporal window of secondary correction and compared between phases in Tjump trials. ${ }^{*}$ : $\mathrm{p}<0.05$. ${ }^{* *}$ : $\mathrm{p}<0.01$. 
Motor responses to target jump in altered force field

775 Footnotes

$776{ }^{1}$ Coriolis force is a pseudo force applied on the whole body in movement in a rotating 777 referential. It increases according to the mass of the segment $(\mathrm{m})$, the rotation velocity $(\omega)$, the 778 segment velocity (v) and the trajectory angle of the displacement $(\theta)$. Formula: $\mathrm{FCor}=2 \mathrm{~m} . \omega . \mathrm{v} / \theta$

779 Centrifugal force is a force applied in a rotating referential. It increases according to the mass 780 of the segment (m), the linear velocity on the tangent to the trajectory (v), the radius of the curve 781 (r). Formula: FCen $=m \cdot v^{2} / r$ 